\section{The relationship between social capital, social support and the adequate use of prenatal care}

\author{
A relação entre capital social e suporte social com \\ a adequação da utilização da atenção pré-natal
}

\author{
1 Escola Nacional de Saúde \\ Pública Sergio Arouca \\ Fundação Oswaldo Cruz, Rio \\ de Janeiro, Brasil. \\ 2 Instituto de Estudos em \\ Saúde Coletiva, Universidade \\ Federal do Rio de Janeiro, Rio \\ de Janeiro, Brasil. \\ Correspondence \\ M. C. Leal \\ Escola Nacional de Saúde \\ Pública Sergio Arouca, \\ Fundação Oswaldo Cruz. \\ Rua Leopoldo Bulhões 1480, \\ Rio de Janeiro, RJ 21041-210 \\ Brasil. \\ duca@fiocruz.br
}

\begin{abstract}
This study investigated the relationship between social capital and social support and the adequate use of prenatal care. A follow-up study involving 1,485 pregnant women was conducted in two cities in the Rio de Janeiro State, Brazil. Demographic and socioeconomic characteristics, social support and social capital data were collected during the first trimester of pregnancy. The postpartum period included information on levels of prenatal care utilization, social networks, parity, obstetric and gestational risk and prenatal care attendance. Hierarchized multinomial logistic regression was used in the statistical analysis. Prenatal care use above adequate levels was associated with high social capital at the city level (aggregated social capital), socioeconomic status and working during pregnancy. Lower non-aggregated contextual and compositional social capital, gestational risk and pattern of prenatal care were associated with inadequate prenatal care utilization. Contextual social capital and social support were found to be social determinants for the appropriate use of prenatal care.
\end{abstract}

Social Inequity; Prenatal Care; Social Support
Maria do Carmo Leal 1

Ana Paula Esteves Pereira 1

Gabriela de Almeida Lamarca 1

Mario Vianna Vettore 2

\section{Introduction}

A satisfactory birth for both fetus and mother depends upon careful monitoring during pregnancy. Screening tests for the early identification of high risk pregnant women are considered the foremost strategies for the prevention of maternal and infant morbidity and mortality 1 . Other strategies developed during prenatal care include clinical management, prophylaxis of diseases, immunization and health promotion practices 2,3 . Prenatal care is a window of opportunity for promoting a healthy lifestyle, including learning how to make healthy food choices, weight control, stopping smoking and reduction of alcohol consumption 4,5.

The Brazilian Ministry of Health (Ministério da Saúde) recommends at least six prenatal care visits during the gestational period: one in the first, two in the second and three in the third trimester of pregnancy ${ }^{6}$. In Rio de Janeiro State, prenatal care coverage involving at least one prenatal visit reached 98 per cent in 2008, which can be considered as universal prenatal coverage (http://www.datasus.gov.br). However, there are discrepancies regarding the number of prenatal visits and the timing of the initiation of care between different cities in the state .

A large survey conducted in Rio de Janeiro city demonstrated that age, educational level, living with the father of the newborn, having fewer children and satisfaction with preg- 
nancy were associated with adequate prenatal care utilization. On the other hand, inadequate prenatal care was significantly higher among adolescents, lighter-skinned black and darkerskinned black mothers, those living in favelas, those without paid work and those who had attempted abortion. Inadequate prenatal care utilization among the lighter-skinned/darkerskinned black mothers varied according to educational level 8 . Similar findings were reported by others 9,10 . During the last decades, more attention has been given to interpersonal relationship networks among people who share common values. This is considered to be a kind of capital, and has been denominated "social capital" 11 . Social capital is typically seen as a part of the societal structure (a contextual approach), while social networks and support are often conceptualized at the level of individuals (a compositional approach) 12,13. It is important to view the "contextual" and "compositional" as being interrelated and not mutually exclusive. Indeed, attributing neighborhood differences to "individual" or "compositional" factors does not necessarily imply the absence of, or lack of important place-based processes 14 . The "individual” factors (e.g. low education, low income) are often themselves a product of the place where people live (places make people) 15. In the same vein, the reverse is also true, that "contextual" factors are rarely independent of the individuals (people make places).

Social capital has an affect on health, by promoting the dissemination and adoption of healthy behaviors, promoting greater access to health services, raising awareness of human rights and promoting self-esteem and mutual respect 16 .

Compositional social capital is related to social support, social networks, diversity, social resources, volume and structure 11 . The importance of compositional social capital in adherence to prenatal care has been highlighted. Social support from the partner was reported as a relevant factor for the adequate use of prenatal care and for the adoption of health related behaviors during pregnancy 17 . Furthermore, women who initiated prenatal care at an early stage were more likely to live with a partner and had more social networks 18 .

Unfavorable social conditions are related to low scores of social capital and this interaction reinforces health inequalities 19 . There are not many Brazilian studies that consider the role of social capital in shaping the outcome of prenatal care. The objective of this study, then, was to investigate the relationship of social capital and social support with adequate prenatal care use in two cities in the Rio de Janeiro State with different socioeconomic conditions and levels of health services.

\section{Methods}

The study was approved by the Ethics Research Committee at the Brazilian National School of Public Health, Oswaldo Cruz Foundation (Escola Nacional de Saúde Pública Sergio Arouca, Fundação Oswaldo Cruz - ENSP/Fiocruz - protocol $n^{\circ}$. 158/06). A follow-up study of pregnant women was conducted in two middle-sized cities in the Rio de Janeiro State. These cities were chosen because of their demographic similarities, differences in social capital and social support proxy measures and for their differences in the provision of prenatal care. The per capita income was $118 \%$ lower while the homicide rate was 10 times higher in the city with the worst social capital proxy measures (city 1) compared with city 2 (http://www.ibge.gov.br; Secretaria de Estado de Segurança Pública do Rio de Janeiro. http://urutau.proderj.rj.gov.br/isp/admin/pa ginas/upboletim/2006_03_bol.pdf, accessed on Jun/2006). The proportion of gynecologists per women between 10-49 years-old was three times less in city 1 than in city 2 . Prenatal care in cities 1 and 2 included 10 and 26 prenatal care units, respectively.

The sample size was established at 1,400 women, considering the prevalence of $25.8 \%$ inadequate and $8.3 \%$ more than adequate prenatal care utilization 8 , with a significance level of $5 \%$ and $95 \%$ power to detect differences of at least $5 \%$. The sample was increased by $20 \%$ due to possible losses during follow-up of pregnant women, resulting in a final sample of 1,680 pregnant women.

Initially, a pilot study with 95 pregnant women randomly selected at the same prenatal care units of the main study was conducted to test the questionnaires. No changes were needed. After that, a test-retest study with a 15 day interval between interviews was conducted with 90 pregnant women to check for internal consistency and the temporal reliability of the scales. The Intraclass Correlation Coeficient between the two interviews was 0.893 for social capital, and ranged between 0.860 (emotional support) and 0.907 (material support) for the social support scale dimensions. Cronbach's $\alpha$ coefficient for social capital was 0.706 and ranged from 0.706 (affectionate support) to 0.863 (emotional support) for social support scale factors.

In the main study, primary data were obtained in the baseline and follow-up from indi- 
vidual standardized interviews performed by 14 trained interviewers.

The selection criteria were women in the first trimester of pregnancy and those who had given birth to a live or stillborn child. Women who experienced miscarriage or abortion (birth weight $<500$ g or gestational weeks $<22$ ) were excluded.

The baseline data collection was performed in 2008, and included pregnant women randomly selected from those who sought prenatal care at the state-funded health care units administered by the Brazilian Unified National Health System (SUS). The participants were gathered at five prenatal care units (primary and referral health centers) in each city, which represent $90 \%$ of prenatal care coverage in both cities.

At the baseline, demographic and socioeconomic characteristics, social support and social capital were collected. The follow-up was performed in the post-partum period immediately after the delivery in the ward of the maternity hospitals or at home within 30 days after discharge.

The data obtained in the follow-up were adequacy of prenatal care utilization, social networks, obstetric history, prenatal attendance and gestational risk.

The assessment of the adequacy of prenatal care utilization was performed using the Kotelchuck index 20 . This index characterizes prenatal care utilization in two independent and distinctive dimensions: adequacy of initiation of prenatal care and adequacy of received services, once the prenatal has begun. In this study, the adequacy of prenatal care initiation and the expected number of visits were based on recommendations from the Brazilian Ministry of Health 6 , adjusted for gestational age at initiation of care and for gestational age at delivery for preterm births. The dimensions were combined into a single summary prenatal care utilization index, which has four categories: inadequate, intermediate, adequate and more than adequate utilization 7,20 . In the present study, the two outcomes were "more than adequate prenatal care utilization" and "inadequate prenatal care utilization".

Social capital is the set of norms and networks that enable people to act collectively 21 . This concept was operationalized from the following indicators: social trust, social control, empowerment, political efficacy and neighborhood security 24 . Social trust refers to the perception of trust in people, inter-relationships and solidarity with the social community neighborhood 16. Social control refers to people's perceptions of community. Social control assesses whether neighbours would intervene in situ- ations where children were engaging in delinquent behaviour 23. Empowerment relates to access to economic and educational resources, information, goods, money and skills that can be used to bring about environmental changes and influence other people and institutions with competing interests or conflicting political perspectives 24 . Political efficacy refers to people's perceptions in relation to political systems and politicians 25 . Neighborhood security refers to the perception of people about safety in the place where live 26 . Social support is a system of formal and informal relationships through which individuals receive emotional support, material or information to cope with stressful emotional situations 27 . Social networks are the "web" of social relationships surrounding the individual as well as their characteristics, or groups of people who have contact with that individual, or with some form of participation 28 . Since each subscale of the social capital questionnaire was made up of different numbers of items, the final scores for each subscale were standardized from 0 to 100 . In this way, the subscales were comparable and could be added up to form the social capital variable.

The social capital questionnaire used was developed and previously tested in the Brazilian population, with adequate internal consistency for the 30 item scale (Cronbach's $\alpha>0.70) 22$.

The questionnaire of social support consists of 19 items comprising five dimensions of functional social support: material, affective, emotional, positive social interaction, and information 29,30 .

The Social Network questionnaire consisted of five questions concerning the person's relationship with their family and friends, and their participation in social groups. The social support and social network questionnaires were found to be reliable for the Brazilian population 30,31.

Social capital and social support data were used to characterize both cities. It was assumed the selected cities would represent different levels of contextual (aggregated and non aggregated) social capital as ecological exposure and social support was used to reflect compositional social capital. Furthermore, non-aggregated social capital and social support were also considered as individual variables.

Covariates were demographic and socioeconomic characteristics, obstetric history, prenatal care attendance and pregnancy risk.

Demographic data were age and race/skin color, which was based on self-perception of skin color.

Socioeconomic characteristics were social class, per capita family income, persons per 
room, years of schooling, work during pregnancy and marital status.

The social class classification used is based on market power comprising a group of specific indicators and level of education of the head of household. A set of points is assigned to these indicators and a final score defines the socioeconomic groups; A (highest), B, C, D, and E (lowest) 32 .

Obstetric history included parity, previous history of fetal or neonatal death, previous low birth weight and previous preterm birth. Prenatal care attendance data were prenatal care in more than one health service and prenatal in more than one municipality. Women were considered at gestational risk when at least one of the following diseases/events was self-reported: diabetes, hypertension, HIV infection, heart diseases, kidney diseases, autoimmune diseases, threatened abortion, previous perinatal death, previous preterm birth or previous low birth weight.

\section{Statistical analysis}

The mean scores of social capital and social support were compared between the two cities with the corresponding $95 \% \mathrm{t}$ test confidence intervals of the difference.

Demographic and socioeconomic characteristics and parity were compared between the two cities through chi-square test. Social support and social network were compared between the categories of covariates for each city. Bivariate analyses of social capital, social support, social network and covariates with adequacy of prenatal care utilization were conducted for each city. Continuous variables were compared using the $t$ test and ANOVA, while categorical variables were analyzed using the chi-square test, with a significance level of $5 \%$.

Multinomial logistic regression was performed to test the association of city, social capital and social support with adequacy of prenatal care utilization, adjusting for covariates. Initially bivariate analysis was performed between independent variables and "more than adequate utilization" and "inadequate utilization". Those variables that present $\mathrm{p} \leq 0.20$ were considered for the multivariate analysis. Since socioeconomic characteristics moderate the effect of social capital and social support on the adequacy of prenatal care utilization, interactions between social capital, social support and socioeconomic characteristics were tested by constructing separate models and adding and removing each interaction term one by one. No interaction was found (results not shown).
Hierarchised multinomial multivariate logistic analysis was performed to evaluate the association of social capital and social support with adequacy of prenatal care utilization, controlling for potential confounders. The first block included the variable "city". The second block was composed of social capital, social support and social network. The third block involved demographic and socioeconomic characteristics, and the fourth block included obstetric history, prenatal care attendance and gestation risk. The significance of the additional variables was tested at each stage and non significant ones $(\mathrm{p}>$ 0.20 ) were eliminated in order to reduce the discrepancy between the data and the model and to find an economic model with relatively few parameters.

In the fully adjusted logistic model we included all the variables that present $\mathrm{p} \leq 0.20$ at the bivariate analysis, independently of whether it was from the same level or not.

The association was estimated through odds ratio with a significance level of $5 \%$. Regression coefficients for social capital and per capita family income were multiplied by 5 and 100 , respectively, on the log scale, so that they indicate a change in the outcome variable for every increase in 5 and 100 units in social capital and per capita family income. All statistical tests were performed with SPSS, version 17.0 (SPSS Inc., Chicago, USA).

\section{Results}

Of the 1,750 pregnant women invited to participate in the study, 70 (4\%) declined. Of the 1,680 women that participated in the baseline, 12 $(0.7 \%)$ did not agree to participate in the followup and 183 (10.9\%) were lost in the follow-up: miscarriage, abortion, moved home or were not found in the follow-up. The final sample involved 1,485 women, which corresponds to $88.4 \%$ of the baseline.

The mean age of the subjects was $24.8 \pm 6.2$ years at baseline, and $34 \%$ were white, $42.5 \%$ were lighter-skinned black and $23.5 \%$ was darkerskinned black. The proportions of per capita family income were $44.3 \%$ ( $\leq 1 / 2$ the minimum wage), $37.9 \%$ (> $1 / 2-\leq 1$ minimum wage) and $17.8 \%$ (> 1 minimum wage). The mean of the mother's schooling was $7.7 \pm 2.9$ years. The frequency of social class B, C, D and E was 5.6, 61.5, 28.2 and 4.8 per cent, respectively.

Table 1 compares social capital and social support scores between the two cities. Social trust, social control, neighborhood security and the total score of social capital were statistically 
Social capital and social support among pregnant women in two cities in Rio de Janeiro State, Brazil, 2008.

\begin{tabular}{|c|c|c|c|c|c|c|c|c|}
\hline & \multicolumn{2}{|c|}{ City 1 (N =722) } & \multicolumn{2}{|c|}{ City $2(\mathrm{~N}=958)$} & \multirow[t]{2}{*}{ Difference } & \multicolumn{2}{|c|}{$\begin{array}{c}95 \% \mathrm{Cl} \text { of the } \\
\text { difference }\end{array}$} & \multirow[t]{2}{*}{ p-value } \\
\hline & Mean & SD & Mean & SD & & Lower & Upper & \\
\hline \multicolumn{9}{|l|}{ Social capital } \\
\hline Social trust & 6.83 & 4.73 & 7.47 & 5.13 & 0.64 & 0.162 & 1.121 & 0.009 \\
\hline Social control & 11.22 & 6.70 & 12.49 & 6.90 & 1.27 & 0.610 & 1.928 & $<0.001$ \\
\hline Empowerment & 3.32 & 3.98 & 2.97 & 3.61 & -0.35 & -0.711 & 0.018 & 0.062 \\
\hline Neighborhood security & 15.11 & 4.09 & 16.07 & 3.89 & 0.96 & 0.575 & 1.343 & $<0.001$ \\
\hline Political efficacy & 1.64 & 3.35 & 1.66 & 3.57 & 0.02 & -0.320 & 0.352 & 0.926 \\
\hline Social capital (global) & 38.13 & 12.71 & 40.67 & 13.14 & 2.54 & 1.286 & 3.791 & $<0.001$ \\
\hline \multicolumn{9}{|l|}{ Social support } \\
\hline Emotional support & 14.52 & 5.36 & 15.70 & 5.05 & 1.18 & 0.680 & 1.682 & $<0.001$ \\
\hline Material support & 14.70 & 5.10 & 15.01 & 5.24 & 0.31 & -0.194 & 0.807 & 0.230 \\
\hline Affective support & 18.33 & 3.07 & 18.64 & 2.69 & 0.31 & 0.038 & 0.591 & 0.026 \\
\hline Positive interaction & 15.64 & 4.86 & 16.52 & 4.53 & 0.88 & 0.424 & 1.328 & $<0.001$ \\
\hline Information support & 14.94 & 5.03 & 15.50 & 5.02 & 0.56 & 0.070 & 1.043 & 0.025 \\
\hline
\end{tabular}

SD: standard deviation; 95\% Cl: 95\% confidence interval.

Note: all dimensions of social capital and social support range from 0 to 20. Social capital (global) ranges from 0 to 100.

lower for women from city 1 than for their counterparts from city 2 . A similar trend was found when comparing the dimensions of social support. City 1 showed lower levels of emotional support, affective support, positive interaction and information support compared with city 2 $(\mathrm{p}<0.05)$.

Demographic and socioeconomic characteristics for the two cities are shown in Table 2. The mean age and the proportion of white women were significantly higher for women from city 2 . Each demographic and socioeconomic characteristic was statistically different between the two cities. Women from city 1 were more likely than those from city 2 to be from a low social class, to report low per capita family income, to be lighterskinned black, to be a teenager, to not work during pregnancy, to be married and to be in their first pregnancy $(\mathrm{p}<0.05)$. They also reported higher schooling $(\mathrm{p}<0.05)$.

Comparative analysis was conducted separately in each city to determine which social support domains were significantly related to demographic and socioeconomic characteristics and parity (Table 2). Socioeconomic status, per capita family income, schooling and parity were associated with all dimensions of social support in both cities $(\mathrm{p}<0.05)$. The proportion of people per room was inversely associated with all dimensions of social support (city 1) and with emotional support (city 2).
Maternal age demonstrated statistical significance with material support in city 1 and emotional support in city 2 . Married women reported higher affective support in both cities, and lower material support (city 1). The scores for positive interaction were higher among those working during pregnancy compared with those not working. Affective support was also associated with work during pregnancy (city 1) (Table 2).

The analyses of social network characteristics according to demographic and socioeconomic covariates for each city are presented in Table 3.

Having a relative to talk to was inversely associated with parity in both cities, and positively associated with schooling (city 2). Women who indicated "having a friend to talk to" reported more years of schooling in both cities, higher per capita family income (city 1 ), working during pregnancy (city 2), to be unmarried (city 2) and with fewer children (city 2$)(\mathrm{p}<0.05)$. Participation in a neighborhood association was more common in older women and those with more children in both cities, in women from higher social classes (city 1), and in women with less years of schooling (city 2).

Married women were more involved in voluntary work (city 2), as well as those from higher social classes (city 1) and with more years of schooling (city 1). Women from a higher social class reported greater participation in religious activities (city 1) and greater per capital family 
Socio-demographic differences between the cities, social support according to socio-demographic characteristics in pregnant women from two cities in Rio de Janeiro State, Brazil, 2008.

\begin{tabular}{|c|c|c|c|c|c|c|c|c|c|c|c|c|c|}
\hline & \multirow{3}{*}{$\begin{array}{c}\text { City } 1 \\
(\mathrm{~N}=722) \\
\mathrm{n}(\%)\end{array}$} & \multirow{3}{*}{$\begin{array}{c}\text { City } 2 \\
(\mathrm{~N}=958) \\
\mathrm{n}(\%)\end{array}$} & \multirow[t]{3}{*}{ p-value } & \multicolumn{10}{|c|}{ City 1} \\
\hline & & & & \multicolumn{2}{|c|}{ Emotional } & \multicolumn{2}{|c|}{ Material } & \multicolumn{2}{|c|}{ Affective } & \multicolumn{2}{|c|}{$\begin{array}{c}\text { Positive } \\
\text { interaction }\end{array}$} & \multicolumn{2}{|c|}{ Information } \\
\hline & & & & Mean & p-value & Mean & p-value & Mean & p-value & Mean & p-value & Mean & $\mathrm{p}$-value \\
\hline \multicolumn{14}{|l|}{ Socioeconomic status } \\
\hline$E+D$ & $272(37.7)$ & $280(29.2)$ & * & 13.6 & * & 13.8 & * & 17.7 & * & 14.7 & * & 14.3 & * \\
\hline$C+B$ & $450(62.3)$ & $678(70.77)$ & & 15.1 & & 15.2 & & 18.7 & & 16.2 & & 15.3 & \\
\hline \multirow{2}{*}{\multicolumn{14}{|c|}{$\begin{array}{l}\text { Per capita family income ** } \\
\text { (minimum wages }{ }^{\star \star \star} \text { ) }\end{array}$}} \\
\hline & & & & & & & & & & & & & \\
\hline$\leq 1 / 2$ & $327(48.9)$ & $318(41.0)$ & * & 13.8 & * & 14.2 & * & 17.8 & * & 14.6 & * & 14.2 & * \\
\hline$>1 / 2$ and $\leq 1$ & $238(35.6)$ & $300(38.7)$ & & 14.9 & & 14.9 & & 18.6 & & 16.5 & & 15.4 & \\
\hline$>1$ & $104(15.5)$ & $157(20.3)$ & & 16.2 & & 16.2 & & 19.3 & & 17.3 & & 16.2 & \\
\hline \multicolumn{14}{|l|}{ People per room } \\
\hline$\leq 2$ & $556(77.0)$ & 790 (82.5) & * & 14.9 & * & 15.0 & * & 18.6 & * & 16.1 & * & 15.2 & * \\
\hline$>2$ & $166(23.0)$ & $168(17.5)$ & & 13.3 & & 14.0 & & 17.4 & & 14.2 & & 14.0 & \\
\hline \multicolumn{14}{|l|}{ Pregnant characteristics } \\
\hline \multicolumn{14}{|l|}{ Race/Skin color } \\
\hline White & $165(22.9)$ & 407 (42.5) & * & 14.6 & NS & 14.6 & NS & 18.31 & NS & 15.7 & NS & 14.8 & NS \\
\hline Lighter-skinned black & $375(51.9)$ & $353(36.8)$ & & 14.8 & & 14.9 & & 18.3 & & 15.5 & & 15.2 & \\
\hline Darker-skinned black & $182(25.2)$ & $198(20.7)$ & & 13.9 & & 14.5 & & 18.4 & & 15.9 & & 14.5 & \\
\hline \multicolumn{14}{|l|}{ Age (years) } \\
\hline$\leq 19$ & $185(25.6)$ & $196(20.5)$ & * & 14.6 & NS & 15.6 & * & 18.5 & NS & 15.8 & NS & 15.1 & NS \\
\hline $20-34$ & $495(68.6)$ & $660(68.9)$ & & 14.6 & & 14.4 & & 18.3 & & 15.6 & & 14.9 & \\
\hline$\geq 35$ & $42(5.8)$ & $102(10.6)$ & & 13.8 & & 14.0 & & 18.0 & & 15.1 & & 14.9 & \\
\hline \multicolumn{14}{|l|}{ Years of schooling } \\
\hline$\leq 4$ & 75 (10.4) & $174(18.2)$ & * & 13.4 & * & 14.2 & NS & 17.0 & * & 13.1 & * & 13.7 & * \\
\hline $5-8$ & 304 (42.1) & $431(45.0)$ & & 14.0 & & 14.5 & & 18.2 & & 15.3 & & 14.6 & \\
\hline$\geq 9$ & $343(47.5)$ & $353(36.8)$ & & 15.2 & & 15.0 & & 18.8 & & 16.5 & & 15.6 & \\
\hline \multicolumn{14}{|l|}{ Work } \\
\hline Yes & $254(35.2)$ & 413 (43.2) & * & 14.8 & NS & 15.0 & NS & 18.6 & NS & 16.3 & * & 15.3 & NS \\
\hline No & $468(64.8)$ & $545(56.8)$ & & 14.4 & & 14.6 & & 18.2 & & 15.3 & & 14.8 & \\
\hline \multicolumn{14}{|l|}{ Marital status } \\
\hline Married & $550(76.2)$ & $683(71.2)$ & * & 14.5 & NS & 14.5 & * & 18.5 & * & 15.7 & * & 14.8 & NS \\
\hline Not married & $172(23.8)$ & 275 (28.8) & & 14.7 & & 15.4 & & 17.8 & & 15.5 & & 15.3 & \\
\hline \multicolumn{14}{|l|}{ Parity \# } \\
\hline First child & $313(47.4)$ & 368 (41.9) & * & 15.3 & * & 15.7 & * & 18.8 & * & 16.4 & * & 15.7 & * \\
\hline $2-3$ & 275 (41.5) & 371 (42.3) & & 14.2 & & 14.3 & & 18.4 & & 15.6 & & 14.7 & \\
\hline$\geq 4$ & 73 (11.0) & 139 (15.8) & & 12.4 & & 12.1 & & 16.6 & & 13.0 & & 12.9 & \\
\hline
\end{tabular}

(continues) 


\begin{tabular}{|c|c|c|c|c|c|c|c|c|c|c|}
\hline & \multicolumn{10}{|c|}{ City 2} \\
\hline & \multicolumn{2}{|c|}{ Emotional } & \multicolumn{2}{|c|}{ Material } & \multicolumn{2}{|c|}{ Affective } & \multicolumn{2}{|c|}{ Positive interaction } & \multicolumn{2}{|c|}{ Information } \\
\hline & Mean & $\mathrm{p}$-value & Mean & p-value & Mean & $p$-value & Mean & p-value & Mean & $\mathrm{p}$-value \\
\hline \multicolumn{11}{|l|}{ Socioeconomic status } \\
\hline$E+D$ & 14.7 & * & 13.9 & * & 18.1 & * & 15.3 & * & 14.4 & * \\
\hline$C+B$ & 16.1 & & 15.5 & & 18.9 & & 17.0 & & 16.0 & \\
\hline \multicolumn{11}{|l|}{$\begin{array}{l}\text { Per capita family income } \\
\text { (minimum wages }{ }^{\star \star \star} \text { ) }\end{array}$} \\
\hline$\leq 1 / 2$ & 14.8 & * & 14.3 & * & 18.1 & * & 15.6 & * & 14.8 & * \\
\hline$>1 / 2$ and $\leq 1$ & 16.1 & & 15.1 & & 19.0 & & 16.9 & & 15.8 & \\
\hline$>1$ & 16.6 & & 15.5 & & 19.1 & & 17.4 & & 16.4 & \\
\hline \multicolumn{11}{|l|}{ People per room } \\
\hline$\leq 2$ & 15.8 & & 15.1 & NS & 18.7 & * & 16.6 & NS & 15.5 & NS \\
\hline$>2$ & 15.4 & & 14.6 & & 18.2 & & 16.0 & & 15.5 & \\
\hline \multicolumn{11}{|l|}{ Pregnant characteristics } \\
\hline \multicolumn{11}{|l|}{ Race/Skin color } \\
\hline White & 15.8 & NS & 15.1 & NS & 18.8 & NS & 16.8 & NS & 15.7 & NS \\
\hline Lighter-skinned black & 15.7 & & 15.0 & & 18.6 & & 16.4 & & 15.6 & \\
\hline Darker-skinned black & 15.5 & & 14.9 & & 18.4 & & 16.1 & & 15.0 & \\
\hline \multicolumn{11}{|l|}{ Age (years) } \\
\hline$\leq 19$ & 16.5 & * & 15.8 & NS & 18.7 & NS & 16.9 & NS & 16.1 & NS \\
\hline $20-34$ & 15.6 & & 14.8 & & 18.6 & & 16.5 & & 15.3 & \\
\hline$\geq 35$ & 15.2 & & 14.8 & & 18.3 & & 16.1 & & 15.5 & \\
\hline \multicolumn{11}{|l|}{ Years of schooling } \\
\hline$\leq 4$ & 14.9 & * & 14.5 & * & 18.1 & * & 15.5 & * & 14.6 & * \\
\hline $5-8$ & 15.6 & & 14.7 & & 18.7 & & 16.6 & & 15.4 & \\
\hline$\geq 9$ & 16.2 & & 15.7 & & 18.9 & & 17.0 & & 16.1 & \\
\hline \multicolumn{11}{|l|}{ Work } \\
\hline Yes & 16.0 & NS & 15.1 & NS & 18.9 & * & 16.9 & * & 15.7 & NS \\
\hline No & 15.5 & & 15.0 & & 18.5 & & 16.2 & & 15.4 & \\
\hline \multicolumn{11}{|l|}{ Marital status } \\
\hline Married & 15.7 & NS & 14.8 & NS & 18.9 & * & 16.7 & NS & 15.5 & NS \\
\hline Not married & 15.7 & & 15.5 & & 18.0 & & 16.1 & & 15.6 & \\
\hline \multicolumn{11}{|l|}{ Parity\# } \\
\hline First child & 16.6 & * & 16.0 & * & 18.8 & * & 17.0 & * & 16.3 & * \\
\hline $2-3$ & 15.2 & & 14.5 & & 18.8 & & 16.6 & & 15.1 & \\
\hline$\geq 4$ & 14.4 & & 13.5 & & 17.8 & & 15.0 & & 14.3 & \\
\hline
\end{tabular}

NS: non significant.

${ }^{*} p<0.05$;

** $N=669$ in city 1 and $N=775$ in city 2;

*** Minimum wage in Rio de Janeiro State in $2008=\mathrm{R} \$ 415.00$ equivalent to US $\$ 230.00$;

\# $N=661$ in city 1 and $N=878$ in city 2 .

Note: all dimensions of social support range from 0 to 20 . 
Social network according to sociodemographic characteristics in pregnant women from two cities in Rio de Janeiro State, Brazil, 2008.

\begin{tabular}{|c|c|c|c|c|c|c|c|c|c|c|c|}
\hline & \multicolumn{11}{|c|}{ City $1(N=661)$} \\
\hline & \multirow[t]{2}{*}{$\mathbf{n}$} & \multicolumn{2}{|c|}{$\begin{array}{c}\text { Have a relative } \\
\text { to talk }\end{array}$} & \multicolumn{2}{|c|}{$\begin{array}{l}\text { Have a friend } \\
\text { to talk }\end{array}$} & \multicolumn{2}{|c|}{$\begin{array}{c}\text { Neghborhood } \\
\text { association }\end{array}$} & \multicolumn{2}{|c|}{$\begin{array}{c}\text { Voluntary work, } \\
\text { charity }\end{array}$} & \multicolumn{2}{|c|}{$\begin{array}{l}\text { Religious } \\
\text { activities }\end{array}$} \\
\hline & & Yes (\%) & $p$-value & Yes (\%) & $\mathrm{p}$-value & Yes (\%) & $\mathrm{p}$-value & Yes (\%) & $\mathrm{p}$-value & Yes (\%) & p-value \\
\hline \multicolumn{12}{|l|}{ Socioeconomic status } \\
\hline$E+D$ & 249 & 77.9 & NS & 52.6 & NS & 3.2 & * & 1.6 & * & 71.9 & * \\
\hline $\mathrm{C}+\mathrm{B}$ & 412 & 81.8 & & 58.3 & & 7.5 & & 4.9 & & 79.9 & \\
\hline \multicolumn{12}{|l|}{ Per capita family income } \\
\hline \multicolumn{12}{|l|}{ (minimum wages) ** } \\
\hline$\leq 1 / 2 * \star *$ & 300 & 78.7 & NS & 50.7 & * & 6.7 & NS & 3.7 & NS & 78.3 & NS \\
\hline$>1 / 2$ and $\leq 1$ & 222 & 79.3 & & 62.6 & & 4.1 & & 4.5 & & 75.7 & \\
\hline$>1$ & 95 & 84.2 & & 64.2 & & 7.4 & & 2.1 & & 73.7 & \\
\hline \multicolumn{12}{|l|}{ People per room } \\
\hline$\leq 2$ & 511 & 80.2 & NS & 56.9 & NS & 5.7 & NS & 3.5 & NS & 76.9 & NS \\
\hline$>2$ & 150 & 80.7 & & 53.3 & & 6.7 & & 4.0 & & 76.7 & \\
\hline \multicolumn{12}{|l|}{ Pregnant characteristics } \\
\hline \multicolumn{12}{|l|}{ Race/Skin color } \\
\hline White & 151 & 80.8 & NS & 62.3 & NS & 6.0 & NS & 3.3 & NS & 76.8 & NS \\
\hline Lighter-skinned black & 339 & 80.2 & & 51.9 & & 4.1 & & 3.5 & & 75.5 & \\
\hline Darker-skinned black & 171 & 80.1 & & 59.1 & & 9.4 & & 4.1 & & 79.5 & \\
\hline \multicolumn{12}{|l|}{ Age (years) } \\
\hline$\leq 19$ & 171 & 52.6 & NS & 3.5 & NS & 2.3 & * & 71.9 & NS & 79.8 & NS \\
\hline $20-34$ & 453 & 57.4 & & 6.8 & & 3.5 & & 77.9 & & 71.4 & \\
\hline$\geq 35$ & 37 & 56.8 & & 5.4 & & 10.8 & & 86.5 & & 82.8 & \\
\hline \multicolumn{12}{|l|}{ Years of schooling } \\
\hline$\leq 4$ & 70 & 71.4 & NS & 50.0 & * & 7.1 & NS & 4.3 & * & 77.1 & NS \\
\hline $5-8$ & 272 & 79.8 & & 49.6 & & 3.7 & & 1.1 & & 76.1 & \\
\hline$\geq 9$ & 319 & 82.8 & & 63.0 & & 7.5 & & 5.6 & & 77.4 & \\
\hline \multicolumn{12}{|l|}{ Work } \\
\hline Yes & 427 & 80.8 & NS & 53.4 & NS & 4.7 & NS & 3.0 & NS & 75.9 & NS \\
\hline No & 234 & 79.5 & & 61.1 & & 8.1 & & 4.7 & & 78.6 & \\
\hline \multicolumn{12}{|l|}{ Marital status } \\
\hline Married & 505 & 79.4 & NS & 56.6 & NS & 5.9 & NS & 3.4 & NS & 77.6 & NS \\
\hline Not married & 156 & 83.3 & & 54.5 & & 5.8 & & 4.5 & & 74.4 & \\
\hline \multicolumn{12}{|l|}{ Parity \# } \\
\hline First child & 313 & 83.8 & * & 59.6 & NS & 3.2 & * & 2.9 & NS & 75.8 & NS \\
\hline $2-3$ & 275 & 80.7 & & 54.9 & & 8.4 & & 4.4 & & 77.1 & \\
\hline$\geq 4$ & 73 & 64.4 & & 45.2 & & 8.2 & & 4.1 & & 80.8 & \\
\hline
\end{tabular}

(continues) 


\begin{tabular}{|c|c|c|c|c|c|c|c|c|c|c|c|}
\hline & \multicolumn{11}{|c|}{ City $2(\mathrm{~N}=887)$} \\
\hline & \multirow[t]{2}{*}{$\mathrm{n}$} & \multicolumn{2}{|c|}{$\begin{array}{c}\text { Have a relative } \\
\text { to talk }\end{array}$} & \multicolumn{2}{|c|}{$\begin{array}{l}\text { Have a friend } \\
\text { to talk }\end{array}$} & \multicolumn{2}{|c|}{$\begin{array}{l}\text { Neghborhood } \\
\text { association }\end{array}$} & \multicolumn{2}{|c|}{$\begin{array}{c}\text { Voluntary work, } \\
\text { charity }\end{array}$} & \multicolumn{2}{|c|}{$\begin{array}{l}\text { Religious } \\
\text { activities }\end{array}$} \\
\hline & & Yes (\%) & p-value & Yes (\%) & $\mathrm{p}$-value & Yes (\%) & p-value & Yes (\%) & p-value & Yes (\%) & $\mathrm{p}$-value \\
\hline \multicolumn{12}{|l|}{ Socioeconomic status } \\
\hline$E+D$ & 255 & 78.4 & NS & 46.3 & * & 3.5 & NS & 2.0 & NS & 58.4 & NS \\
\hline $\mathrm{C}+\mathrm{B}$ & 623 & 82.5 & & 62.2 & & 4.3 & & 4.2 & & 60.9 & \\
\hline \multicolumn{12}{|l|}{ Per capita family income } \\
\hline \multicolumn{12}{|l|}{ (minimum wages) ${ }^{\star \star}$} \\
\hline$\leq 1 / 2 * \star \star$ & 286 & 79.0 & NS & 55.2 & NS & 4.9 & NS & 4.9 & NS & 52.8 & * \\
\hline$>1 / 2$ and $\leq 1$ & 282 & 83.7 & & 60.8 & & 4.2 & & 2.5 & & 62.9 & \\
\hline$>1$ & 143 & 81.1 & & 64.3 & & 2.8 & & 3.5 & & 69.9 & \\
\hline \multicolumn{12}{|l|}{ People per room } \\
\hline$\leq 2$ & 717 & 81.7 & NS & 58.9 & NS & 3.9 & NS & 3.9 & NS & 61.4 & NS \\
\hline$>2$ & 161 & 79.5 & & 51.6 & & 5.0 & & 1.9 & & 54.7 & \\
\hline \multicolumn{12}{|l|}{ Pregnant characteristics } \\
\hline \multicolumn{12}{|l|}{ Race/Skin color } \\
\hline White & 369 & 83.5 & NS & 59.2 & NS & 2.7 & NS & 3.2 & NS & 60.3 & NS \\
\hline Lighter-skinned black & 319 & 80.9 & & 57.7 & & 5.3 & & 4.7 & & 63.3 & \\
\hline Darker-skinned black & 190 & 77.9 & & 54.2 & & 4.7 & & 2.1 & & 54.7 & \\
\hline \multicolumn{12}{|l|}{ Age (years) } \\
\hline$\leq 19$ & 180 & 82.1 & NS & 56.1 & NS & 2.8 & * & 1.1 & NS & 53.3 & NS \\
\hline $20-34$ & 604 & 82.0 & & 57.9 & & 3.6 & & 3.8 & & 62.0 & \\
\hline$\geq 35$ & 94 & 75.5 & & 58.5 & & 9.6 & & 6.4 & & 61.7 & \\
\hline \multicolumn{12}{|l|}{ Years of schooling } \\
\hline$\leq 4$ & 153 & 78.4 & * & 44.4 & * & 7.8 & * & 5.2 & NS & 54.9 & NS \\
\hline $5-8$ & 404 & 78.7 & & 55.6 & & 4.2 & & 2.5 & & 60.2 & \\
\hline$\geq 9$ & 321 & 86.0 & & 66.4 & & 2.2 & & 4.0 & & 62.6 & \\
\hline \multicolumn{12}{|l|}{ Work } \\
\hline Yes & 491 & 81.5 & NS & 54.1 & * & 3.9 & NS & 3.0 & NS & 56.7 & * \\
\hline No & 387 & 81.1 & & 62.0 & & 4.4 & & 4.1 & & 64.6 & \\
\hline \multicolumn{12}{|l|}{ Marital status } \\
\hline Married & 627 & 81.5 & NS & 54.9 & * & 4.3 & NS & 4.5 & * & 62.7 & * \\
\hline Not married & 251 & 80.9 & & 64.1 & & 3.6 & & 1.2 & & 53.8 & \\
\hline \multicolumn{12}{|l|}{ Parity \# } \\
\hline First child & 367 & 84.5 & * & 62.2 & NS & 2.2 & * & 2.7 & NS & 60.1 & NS \\
\hline $2-3$ & 372 & 82.0 & & 54.8 & & 4.3 & & 3.5 & & 61.3 & \\
\hline$\geq 4$ & 139 & 71.2 & & 52.5 & & 8.6 & & 5.8 & & 57.6 & \\
\hline
\end{tabular}

NS: non significant.

* $\mathrm{p}$-value < 0.05;

** $\mathrm{N}=617$ in city 1 and $\mathrm{N}=711$ in city 2;

*** Minimum wage in Rio de Janeiro State in 2008: $R \$ 415,00$; equivalent to US $\$ 230,00$. 
income (city 2). In addition, the frequency of participation in religious activities was statistically higher among women that worked during pregnancy and married women (city 2) (Table 3).

The association of social capital, demographic and socioeconomic characteristics, obstetric history and prenatal care attendance with adequate prenatal care use in each city is presented in Table 4. Women who received adequate prenatal care presented higher scores for emotional support and positive interaction compared with women who received inadequate prenatal care. The average scores of these social support dimensions were statistically higher for women with more than adequate prenatal attendance than for women with just adequate prenatal care. There were statistical differences in per capita family income, persons per room, parity, years of schooling, working during pregnancy and having prenatal care in more than one health care unit among groups of adequate prenatal care use in both cities $(\mathrm{p}<0.05)$. Women with obstetric and gestational risk were more frequent among those with inadequate prenatal care in city 1 . In addition, in city 1 , there was a significant difference between women who reported prenatal care in more than one municipality and those who did not in the adequate use of prenatal care. Older women groups tended to have a higher proportion of adequate prenatal care and unmarried women were more prone to inadequate prenatal care compared with married women in city 2 (Table 4).

Table 5 shows the bivariate, hierarchical and fully adjusted multinomial analyses. The bivariate multinomial analyses revealed that women living in city 2 (aggregated contextual social capital), those with higher levels of non-aggregated social capital, social control and neighborhood security were more likely to get more than adequate prenatal care. Two dimensions of social support greatly increased the odds of receiving more than adequate prenatal care. Higher emotional support and positive interaction increased $16 \%$ and $14 \%$ the likelihood of receiving more than adequate prenatal care. No social network dimensions reached statistical significance with more than adequate prenatal care. Otherwise, almost all demographic and socioeconomic factors were associated with having more than adequate prenatal care. For example, high socioeconomic status, high per capita family income, lighter skin color, older women and having paid work predicted more than adequate prenatal care.

Factors associated with inadequate prenatal care at the bivariate analysis were lower non-aggregated contextual social capital, lower neighborhood security and lower social support concerning emotional and affective support and positive interaction indicators. In addition, we found that women with higher affective support were less likely to have inadequate prenatal care ( $24 \%$ less for an increase of 5 points in a 0 to 20 scale). Three socioeconomic factors that were positively associated with more than adequate prenatal care associated inversely with inadequate prenatal care: socioeconomic status, per capita family income and paid work, while higher parity and lower schooling only influenced the chance of having inadequate prenatal care. Moreover we found that women at gestational risk, which were supposed to start the prenatal care earlier and carry out more visits, were the ones that had a higher chance of having inadequate prenatal care $(\mathrm{OR}=1.68)$. As expected, the ones that consulted more than one health unit were $60 \%$ less likely to be classified as "inadequate".

Aggregated social capital (city level) increased by eight-fold the odds of more than adequate prenatal care (Table 5). The social variables (level 2) that in the univariate analysis were associated with more than adequate prenatal care lose their statistical significance. However, higher socioeconomic status and having paid work remained statistically significant. The same was true for years of schooling, where the chance of having more than adequate prenatal care increased by almost 7\% for each additional year. Age and skin color were no longer significant.

Higher non-aggregated contextual social capital, neighborhood security, emotional support, affective support and positive interaction remained inversely associated with inadequate prenatal care, even after controlling for the aggregated social capital (hierarchically adjusted). Women with a higher non-aggregate social capital score performed $5.5 \%$ less for inadequate prenatal care for every 5 point increase on a 0 to 100 scale. For the others, the protection was $17.5 \%$, $16.7 \%, 24.3 \%$ and $17.9 \%$ for each 5 point increase on a 0 to 20 scale, respectively.

The strong connection between socioeconomic conditions, social capital and social support, at the individual level, was responsible for the weakness of the association between non-aggregate contextual social capital and inadequacy of prenatal care in the fully adjusted model. Although the direction remained the same, the sample used here did not have sufficient power to detect an independent association.

\section{Discussion}

In this study, contextual social capital (aggregate and non-aggregate) and social support (compo- 
Adequacy of prenatal care according to sociodemographic characteristics in pregnant women from two cities in Rio de Janeiro State, Brazil, 2008.

\begin{tabular}{|c|c|c|c|c|c|c|c|c|c|c|}
\hline & \multicolumn{5}{|c|}{ City $1(\mathrm{~N}=632)$} & \multicolumn{5}{|c|}{ City $2(N=853)$} \\
\hline & $\mathrm{n}$ & $\begin{array}{l}\text { Inadequate } \\
(n=234)\end{array}$ & $\begin{array}{l}\text { Adequate } \\
(n=292)\end{array}$ & $\begin{array}{l}\text { More than } \\
\text { adequate } \\
(n=106)\end{array}$ & $\mathrm{p}$-value & $\mathrm{n}$ & $\begin{array}{l}\text { Inadequate } \\
(n=167)\end{array}$ & $\begin{array}{l}\text { Adequate } \\
(n=203)\end{array}$ & $\begin{array}{l}\text { More than } \\
\text { adequate } \\
(n=483)\end{array}$ & $\mathrm{p}$-value \\
\hline Total (\%) & 632 & 37.0 & 46.3 & 16.7 & - & 853 & 19.6 & 23.8 & 56.6 & - \\
\hline Social Capital (mean) & 632 & 36.8 & 38.2 & 39.0 & 0.240 & 853 & 38.8 & 40.9 & 41.3 & 0.095 \\
\hline \multicolumn{11}{|l|}{ Social Support (mean) } \\
\hline Emotional support & 632 & 13.9 & 14.8 & 15.6 & 0.017 & 853 & 14.7 & 15.6 & 16.0 & 0.023 \\
\hline Material support & 632 & 14.5 & 14.8 & 15.4 & 0.343 & 853 & 14.5 & 15.2 & 14.9 & 0.411 \\
\hline Affective support & 632 & 18.2 & 18.5 & 18.8 & 0.165 & 853 & 18.3 & 18.7 & 18.7 & 0.195 \\
\hline Positive interaction & 632 & 15.1 & 16.1 & 16.3 & 0.029 & 853 & 15.8 & 16.3 & 16.8 & 0.044 \\
\hline Information support & 632 & 14.9 & 15.0 & 15.6 & 0.454 & 853 & 14.8 & 15.5 & 15.6 & 0.247 \\
\hline \multicolumn{11}{|l|}{ Social network (\% of yes) } \\
\hline Have a relative to talk & 633 & 76.1 & 81.6 & 86.8 & 0.055 & 853 & 81.4 & 78.8 & 82.0 & 0.629 \\
\hline Have a friend to talk & 633 & 57.7 & 53.2 & 62.3 & 0.244 & 853 & 52.7 & 57.6 & 59.8 & 0.273 \\
\hline Neghborhood association & 633 & 7.7 & 4.8 & 3.8 & 0.232 & 853 & 6.6 & 3.0 & 3.9 & 0.201 \\
\hline Voluntary work, charity & 633 & 3.8 & 3.1 & 2.8 & 0.845 & 853 & 2.4 & 2.0 & 4.3 & 0.213 \\
\hline Religious activities & 633 & 74.4 & 77.1 & 81.1 & 0.385 & 853 & 50.9 & 59.6 & 63.1 & 0.021 \\
\hline \multicolumn{11}{|l|}{ Socioeconomic status } \\
\hline$E+D$ & 235 & 46.1 & 46.4 & 7.5 & $<0.001$ & 255 & 25.9 & 26.3 & 47.8 & 0.001 \\
\hline $\mathrm{C}+\mathrm{B}$ & 397 & 31.7 & 46.1 & 22.2 & & 598 & 16.9 & 22.7 & 60.4 & \\
\hline \multicolumn{11}{|l|}{$\begin{array}{l}\text { Per capita family income } \\
\text { (minimum wages) * }\end{array}$} \\
\hline$\leq 1 / 2 * \star$ & 288 & 41.7 & 45.5 & 12.8 & $<0.001$ & 280 & 28.2 & 22.9 & 48.9 & $<0.001$ \\
\hline$>1 / 2$ and $\leq 1$ & 210 & 30.5 & 53.8 & 15.7 & & 276 & 12.7 & 22.8 & 64.5 & \\
\hline$>1$ & 92 & 33.7 & 34.8 & 31.5 & & 136 & 9.6 & 19.8 & 70.6 & \\
\hline \multicolumn{11}{|l|}{ People per room } \\
\hline$\leq 2$ & 490 & 33.0 & 48.0 & 19.0 & $<0.001$ & 696 & 17.7 & 24.1 & 58.2 & 0.012 \\
\hline$>2$ & 142 & 50.7 & 40.1 & 9.2 & & 157 & 28.0 & 22.3 & 49.7 & \\
\hline \multicolumn{11}{|l|}{ Race/skin color } \\
\hline White & 145 & 35.9 & 45.5 & 18.6 & 0.871 & 359 & 16.2 & 23.4 & 60.4 & 0.166 \\
\hline Lighter-skinned black & 324 & 38.5 & 45.1 & 16.4 & & 307 & 21.2 & 23.1 & 55.7 & \\
\hline Darker-skinned black & 163 & 35.0 & 49.0 & 16.0 & & 187 & 23.5 & 25.7 & 50.8 & \\
\hline \multicolumn{11}{|l|}{ Age (years) } \\
\hline$\leq 19$ & 167 & 41.3 & 44.9 & 13.8 & 0.128 & 588 & 27.7 & 20.3 & 52.0 & 0.041 \\
\hline $20-34$ & 431 & 34.8 & 48.0 & 17.2 & & 177 & 17.9 & 24.6 & 57.5 & \\
\hline$\geq 35$ & 34 & 44.1 & 29.4 & 26.5 & & 88 & 14.8 & 25.0 & 60.2 & \\
\hline \multicolumn{11}{|l|}{ Years of schooling } \\
\hline$\leq 4$ & 67 & 43.2 & 47.8 & 9.0 & $<0.001$ & 149 & 26.8 & 24.8 & 48.4 & 0.013 \\
\hline $5-8$ & 260 & 44.3 & 46.5 & 9.2 & & 389 & 21.1 & 23.7 & 55.2 & \\
\hline$\geq 9$ & 305 & 29.5 & 45.6 & 24.9 & & 315 & 14.3 & 23.5 & 62.2 & \\
\hline \multicolumn{11}{|l|}{ Employed } \\
\hline No & 405 & 42.0 & 46.2 & 11.8 & $<0.001$ & 482 & 24.7 & 24.9 & 50.4 & $<0.001$ \\
\hline Yes & 227 & 28.2 & 46.3 & 25.5 & & 371 & 12.9 & 22.4 & 64.7 & \\
\hline \multicolumn{11}{|l|}{ Marital status } \\
\hline Married & 481 & 36.3 & 46.2 & 17.5 & 0.670 & 606 & 17.2 & 23.9 & 58.9 & 0.017 \\
\hline Not married & 151 & 39.1 & 46.3 & 14.6 & & 247 & 25.5 & 23.5 & 51.0 & \\
\hline
\end{tabular}

(continues) 


\begin{tabular}{|c|c|c|c|c|c|c|c|c|c|c|}
\hline & \multicolumn{5}{|c|}{ City $1(\mathrm{~N}=632)$} & \multicolumn{5}{|c|}{ City $2(N=853)$} \\
\hline & $\mathbf{n}$ & $\begin{array}{l}\text { Inadequate } \\
\qquad(n=234)\end{array}$ & $\begin{array}{l}\text { Adequate } \\
(n=292)\end{array}$ & $\begin{array}{l}\text { More than } \\
\text { adequate } \\
(n=106)\end{array}$ & p-value & $\mathbf{n}$ & $\begin{array}{l}\text { Inadequate } \\
\qquad(n=167)\end{array}$ & $\begin{array}{l}\text { Adequate } \\
(n=203)\end{array}$ & $\begin{array}{l}\text { More than } \\
\text { adequate } \\
(n=483)\end{array}$ & $\mathrm{p}$-value \\
\hline \multicolumn{11}{|l|}{ Parity } \\
\hline First child & 299 & 33.8 & 45.8 & 20.4 & 0.016 & 357 & 17.9 & 20.2 & 61.9 & 0.027 \\
\hline $2-3$ & 267 & 36.7 & 48.3 & 15.0 & & 359 & 18.7 & 27.0 & 54.3 & \\
\hline$\geq 4$ & 67 & 52.2 & 40.3 & 7.5 & & 137 & 26.3 & 24.8 & 48.9 & \\
\hline \multicolumn{11}{|c|}{$\begin{array}{l}\text { Previous history of fetal or } \\
\text { neonatal death, low birth } \\
\text { weight, prematurity }\end{array}$} \\
\hline No & 543 & 36.5 & 45.3 & 18.2 & 0.047 & 705 & 18.8 & 24.0 & 57.2 & 0.518 \\
\hline Yes & 90 & 40.0 & 52.2 & 7.8 & & 148 & 23.0 & 23.0 & 54.0 & \\
\hline \multicolumn{11}{|c|}{ 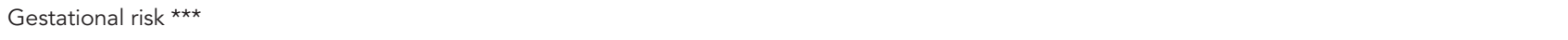 } \\
\hline No & 502 & 33.5 & 48.8 & 17.7 & 0.002 & 693 & 19.5 & 25.0 & 55.5 & 0.238 \\
\hline Yes & 131 & 50.4 & 36.6 & 13.0 & & 160 & 20.0 & 18.7 & 61.3 & \\
\hline \multicolumn{11}{|c|}{$\begin{array}{l}\text { Perinatal care in more than } \\
\text { one health service \# }\end{array}$} \\
\hline No & 369 & 43.1 & 46.9 & 10.0 & $<0.001$ & 706 & 22.0 & 23.9 & 54.1 & 0.001 \\
\hline Yes & 264 & 28.4 & 45.5 & 26.1 & & 125 & 8.0 & 22.4 & 69.6 & \\
\hline \multicolumn{11}{|c|}{$\begin{array}{l}\text { Perinatal care in more than } \\
\text { one municipality }\end{array}$} \\
\hline No & 417 & 41.2 & 47.3 & 11.5 & $<0.001$ & 851 & - & - & - & - \\
\hline Yes & 215 & 28.4 & 44.6 & 27.0 & & 2 & - & - & - & \\
\hline
\end{tabular}

* $N=692$ in city 1 and $N=590$ in city 2 ;

** Minimum wage in Rio de Janeiro State in 2008: $\mathrm{R} \$ 415,00$; equivalent to US $\$ 230,00$;

*** Diabetes, Hypertension, HIV, heart or kidney diseases;

$\# \mathrm{~N}=831$.

sitional social capital) were associated with adequacy of prenatal care use. However, aggregated contextual social capital was measured only in two cities, and other environmental exposures could have been responsible for this finding, like the greater availability and accessibility of prenatal care units in city 2 .

Two main findings on social capital must be underlined because of the two different perspectives used to understand the concept: contextual social capital from Putnam's theory 13 and compositional social capital from Bordieu's theory 12 . On the one hand, more than adequate use of prenatal care was associated with high social capital at the city level (aggregated social capital). On the other hand, the odds of inadequate prenatal care use was higher among women with lower nonaggregated contextual and compositional social capital.

Social capital is a collective attribute of communities or societies, and its beneficial properties are associated with individuals and their social relationships 11 . Poorting 33 used data from 22 European countries to show that rather than having a contextual influence on health, the beneficial properties of social capital can be found at the individual level. Individual levels of social trust and civic participation were strongly associated with self-rated health. At the same time, the aggregate social trust and civic participation variables at the national level were not related to people's subjective health after controlling for compositional differences in sociodemographics.

Putnam's social capital concepts 13 were extended to the functioning of the number of prenatal care units. The possible explanatory pathway that can be considered for the potential contextual aggregated effect of social capital on more than adequate use of prenatal care is that women living in the city with high levels of social capital were more able to overcome individual difficulties in attending prenatal care, such as financial limitations or lack of familial support. Therefore, it seems that those living in communities with high aggregate social capital and with a greater 
Hierarchized multinomial logistic regression between social capital and social support with adequate prenatal care utilization.

\begin{tabular}{|c|c|c|c|c|c|c|c|c|c|c|c|c|}
\hline \multirow[t]{3}{*}{ Variable } & \multicolumn{6}{|c|}{ Adequate vs. more than adequate } & \multicolumn{6}{|c|}{ Adequate vs. inadequate } \\
\hline & \multicolumn{2}{|c|}{ Crude } & \multicolumn{2}{|c|}{$\begin{array}{l}\text { Hierarchically } \\
\text { adjusted }\end{array}$} & \multicolumn{2}{|c|}{ Fully adjusted } & \multicolumn{2}{|c|}{ Crude } & \multicolumn{2}{|c|}{$\begin{array}{l}\text { Hierarchically } \\
\text { adjusted }\end{array}$} & \multicolumn{2}{|c|}{ Fully adjusted } \\
\hline & OR & p-value & OR & p-value & OR & p-value & OR & p-value & OR & p-value & OR & $\mathrm{p}$-value \\
\hline \multicolumn{13}{|l|}{ Level 1} \\
\hline City (reference category $=1$ ) & 6.58 & $<0.001$ & 6.58 & $<0.001$ & 8.07 & $<0.001$ & 1.03 & 0.828 & 1.03 & 0.828 & 0.92 & 0.607 \\
\hline \multicolumn{13}{|l|}{ Level 2} \\
\hline Social capital * (divided by 5) & 1.05 & 0.054 & 1.02 & 0.516 & 0.97 & 0.374 & 0.95 & 0.045 & 0.95 & 0.041 & 0.95 & 0.095 \\
\hline Social trust & 1.05 & 0.473 & - & - & - & - & 0.95 & 0.427 & - & - & - & - \\
\hline Social control & 1.10 & 0.030 & 1.06 & 0.251 & & & 0.93 & 0.115 & 0.92 & 0.108 & 0.95 & 0.375 \\
\hline Empowerment & 0.99 & 0.932 & - & - & - & - & 0.97 & 0.749 & - & - & - & - \\
\hline Neighborhood security & 1.16 & 0.058 & 1.04 & 0.669 & & & 0.86 & 0.055 & 0.85 & 0.049 & 0.85 & 0.071 \\
\hline Political efficacy & 0.99 & 0.912 & - & - & - & - & 0.95 & 0.576 & - & - & - & - \\
\hline \multicolumn{13}{|l|}{ Social support * (divided by 5) } \\
\hline Emotional support & 1.16 & 0.014 & 1.10 & 0.146 & 1.07 & 0.579 & 0.86 & 0.014 & 0.86 & 0.013 & 0.90 & 0.299 \\
\hline Material support & 1.01 & 0.856 & - & - & - & - & 0.92 & 0.204 & 0.92 & 0.199 & 1.03 & 0.736 \\
\hline Affective support & 1.07 & 0.547 & - & - & - & - & 0.80 & 0.056 & 0.80 & 0.056 & 1.04 & 0.845 \\
\hline Positive interaction & 1.14 & 0.054 & 1.09 & 0.226 & 1.01 & 0.904 & 0.85 & 0.019 & 0.85 & 0.018 & 1.00 & 0.968 \\
\hline Information support & 1.10 & 0.140 & 1.08 & 0.257 & 1.06 & 0.663 & 0.95 & 0.433 & - & - & - & - \\
\hline \multirow{2}{*}{\multicolumn{13}{|c|}{$\begin{array}{l}\text { Social network } \\
\text { (reference category = no) }\end{array}$}} \\
\hline & & & & & & & & & & & & \\
\hline Have a relative to talk to & 1.17 & 0.313 & - & - & - & - & 0.88 & 0.430 & - & - & - & - \\
\hline Have a friend to talk to & 1.24 & 0.082 & 1.25 & 0.091 & 1.02 & 0.912 & 1.02 & 0.864 & - & - & - & - \\
\hline Neghborhood association & 0.97 & 0.915 & - & - & - & - & 1.86 & 0.039 & 1.86 & 0.038 & 2.23 & 0.016 \\
\hline Voluntary work, charity & 1.58 & 0.192 & 1.68 & 0.168 & 1.17 & 0.703 & 1.25 & 0.582 & - & - & - & - \\
\hline Religious activities & 0.85 & 0.209 & - & - & - & - & 0.78 & 0.088 & 0.78 & 0.089 & 0.87 & 0.379 \\
\hline \multicolumn{13}{|l|}{ Level 3} \\
\hline Socioeconomic status * & 1.07 & $<0.001$ & 1.05 & 0.015 & 1.08 & 0.002 & 0.96 & 0.003 & 0.99 & 0.746 & 0.99 & 0.635 \\
\hline $\begin{array}{l}\text { Per capita family income * } \\
\text { (divided by } R \$ 100,00 \text { ) }\end{array}$ & 1.14 & $<0.001$ & 1.04 & 0.259 & 1.05 & 0.253 & 0.86 & 0.001 & 0.97 & 0.464 & 0.97 & 0.480 \\
\hline $\begin{array}{l}\text { Race/Skin color ** (reference } \\
\text { category }=\text { darker-skinned black) }\end{array}$ & 1.31 & 0.001 & 0.99 & 0.888 & 1.00 & 0.980 & 0.96 & 0.689 & - & - & - & - \\
\hline Age * & 1.03 & 0.002 & 1.02 & 0.091 & 1.03 & 0.129 & 0.98 & 0.082 & 0.97 & 0.099 & 0.97 & 0.057 \\
\hline Years of schooling * & 1.04 & 0.070 & 1.07 & 0.026 & 1.04 & 0.291 & 0.93 & 0.001 & 0.97 & 0.288 & 0.98 & 0.451 \\
\hline Work (reference category = no) & 1.67 & $<0,000$ & 1.42 & 0.026 & 1.62 & 0.019 & 0.63 & 0.002 & 0.82 & 0.234 & 0.82 & 0.242 \\
\hline Married (reference category = no) & 1.04 & 0.783 & - & - & - & - & 0.80 & 0.130 & 0.73 & 0.077 & 0.72 & 0.068 \\
\hline Parity ** $(1,2,3 \ldots)$ & 0.96 & 0.424 & - & - & - & - & 1.11 & 0.027 & 1.14 & 0.061 & 1.15 & 0.060 \\
\hline \multicolumn{13}{|l|}{ Level 4} \\
\hline $\begin{array}{l}\text { Previous History of fetal or neonatal } \\
\text { death, low birth weight, prematurity } \\
\text { (reference category = no) }\end{array}$ & 0.89 & 0.479 & - & - & - & - & 1.08 & 0.654 & - & - & - & - \\
\hline $\begin{array}{l}\text { Gestational risk } \star \star \star \\
\text { (reference category }=\text { no) }\end{array}$ & 1.30 & 0.104 & 1.34 & 0.238 & 1.34 & 0.238 & 1.73 & 0.001 & 1.69 & 0.005 & 1.69 & 0.005 \\
\hline $\begin{array}{l}\text { Perinatal care in more than one health } \\
\text { service (reference category }=\text { no) }\end{array}$ & 0.860 & 0.27 & - & - & - & - & 0.63 & 0.003 & 0.63 & 0.011 & 0.63 & 0.011 \\
\hline
\end{tabular}

* Continuous variable;

** Ordinal variable;

*** Diabetes, hypertension, HIV, heart or kidney disease. 
availability of health services are more prone to receive more than adequate prenatal care.

This study was not able to examine the direct mechanisms by which contextual social capital may improve access to prenatal care. However, social capital refers to three interdependent community factors, namely interpersonal trust, civic engagement (i.e. active participation in public affairs), and norms of reciprocity (i.e. generalized expectations of cooperative behavior) 13 . Furthermore, social capital may operate to create more humane, efficient, better coordinated, broader, or deeper health care systems. If we extend this reasoning to health care institutions, we may argue that social capital improves access in the same manner that social capital improves local government functioning ${ }^{34}$. Social capital may be the element of values and sense of community that operates to improve both the probability and impact of accountability mechanisms because in higher social capital communities, reputations matter, and shared values and community goals are more likely to exist. Conversely, in low social capital communities, common values and goals are lacking, and reputations do not travel through the community because its residents are more disengaged, resulting in weakened accountability mechanisms 34 .

Socioeconomic status and having paid employment also remained associated with more than adequate utilization of adequate prenatal care in multivariate analysis, showing that individual social conditions were independently associated with prenatal care. Other authors demonstrated the inverse care law in women's health care utilization 35,36 . The use of adequate health services was correlated with having a higher educational level, having private health insurance and being married 35 . In addition, having received a mammogram in the previous two years was associated with urban residence, years of schooling, family income, number of physician visits, and health plan coverage 36 .

Social support components such as emotional support, affective support and positive interaction were inversely associated with inadequate prenatal care. This finding is consistent with the study by St Clair et al. 37 , who reported that utilization of prenatal care was significantly associated with social network structural variables, including size of relative network, emotional intimacy with relatives, frequency of contact with friends and dispersion of friendship network.

Non-aggregated contextual social capital and lower compositional social capital (social support) were associated with inadequate prenatal care use. This occurred independently of aggregate social capital. In this case, the importance of social capital was found at both contextual and compositional levels. According to Fukuyama 38, social capital can be embodied in the smallest and most basic of groups, the family, as well as the largest of all groups, the nation. So, it can be argued that the benefits that individuals accrue from their own social networks can lead to a decrease in inadequate prenatal care use.

It can be assumed that women tended to use informal and specific social relationships (e.g. neighbors, friends and family members) at a minimum cost, giving access to specific sets of resources 11 . This may represent a kind of individual social capital and networks to capitalize benefits towards health, and to overcome contextual barriers to obtain access to prenatal care, like limited availability of health services.

Women who reported participation in neighborhood associations were more prone to inadequate prenatal care use. These women were older, had lower schooling levels and higher parity when compared with those who did not participate in neighborhood associations. Furthermore, schooling and lower parity were associated with more relationships with family and friends. Similarly, St Clair et al. 37 showed that women with higher parity and lower levels of education reported greater participation in neighborhood social networks. In our study, the social networks of women with inadequate prenatal care were characterized by low levels of diversity, and included people from the same social group with similar patterns of health service use.

The social support measures confirmed that pregnant women living in unfavorable social conditions showed lower scores for non-aggregated social capital. In addition, the city with the highest levels of social capital also presented better social indicators. However, the proportion of unmarried women and higher parity were higher in the city with higher social capital. One potential explanation for the unexpected higher parity in that city can be the lower proportion of young people and migrants compared with the city with low levels of social capital, which was constituted as a city 19 years ago, in contrast to the nearly 200 years of existence of the city with high social capital. The higher frequency of women without partners in the city with high social capital may not represent an indicator for social disadvantage, but rather can be understood as a phenomenon of contemporary urban society and the economic independence of women, like female headed households, as shown by Marin \& Piccinini 39 . In the city with high social capital, women who did not live with a partner had more schooling than those who lived with a partner, different to the city with low social capital (data not shown). 
In both cities, high social support was associated with better socioeconomic conditions and formal employment, while the age and parity as well as household crowding were related with lower scores for social support. Married women reported feeling most loved and important to other people but relied less on social support when needed (for example, when sick or needing to go to the doctor). The contrast regarding the perception of material and emotional support among adolescents who frequently lived with supportive family members was striking. The frequency of adolescents was higher in the city with low social capital, and unmarried women lived in households with more people (data not shown).

Regarding the provision of health services, they failed to ensure a better adherence to prenatal health care among women at previous or current obstetric risk, and this finding is in agreement with the Leal et al. 8 study. One hypothesis is that health care systems in the cities in the study were incapable of guaranteeing inter-sectoral referrals to improve the use of prenatal care. The lack of a connection between associative mechanisms for local communities (neighborhood associations) and health care systems to promote the improvement of the use of prenatal services in these communities may be an explanation.
The main positive aspect of this study was its longitudinal design. Measures for social capital and social support were collected during the first trimester of pregnancy while the information about prenatal care use was gathered during the post partum period. The use of adequate proxy indicators for income and violence to select the cities with different levels of social capital was confirmed through the significant differences found between the cities for social capital measures. Furthermore, appropriate questionnaires were used to measure social capital and social support, which assured the validity of the study. Very good psychometric properties were observed in both questionnaires despite the fact the sample included pregnant women, a period of life marked by intense emotional experiences related to maternity.

The present study demonstrated that contextual social capital and social support were social determinants for adequate prenatal care use. In spite of the strong connection between socioeconomic conditions, social capital and social support, independent connections were observed between different social determinants of health and prenatal care, even in cities with extremely poor health service provision.

\section{Resumo}

O objetivo deste estudo foi investigar a associação entre capital social e apoio social com a adequação da atenção pré-natal. Um estudo de seguimento que envolveu 1.485 gestantes foi realizado em duas cidades do Estado do Rio de Janeiro, Brasil. Características demográficas e socioeconômicas e dados de capital social e apoio social foram coletados no 1o trimestre da gestação. No pós-parto, registraram-se informações sobre adequação do pré-natal, redes sociais, risco obstétrico e gestacional e padrão de atendimento pré-natal. Regressão logística hierarquizada multinomial foi utilizada na análise. A utilização mais que adequada do pré-natal foi associada com maior capital social em nível de cidade (capital social agregado), status socioeconômico e trabalho durante a gravidez. Baixo capital social contextual não agregado e composicional, gestação de risco e padrão do uso do pré-natal foram associados com a utilização inadequada do pré-natal. O capital social contextual e o apoio social foram determinantes sociais para a adequação da utilização do pré-natal.

Iniquidade Social; Cuidado Pré-Natal; Apoio Social 


\section{Contributors}

M. C. Leal was responsible for designing and carrying out the research and writing up the article. A. P. E. Pereira participated in the research, data analysis and article write-up. G. A. Lamarca contributed in the research and in writing the article. M. V. Vettore was involved in carrying out the research and writing up the article.

\section{References}

1. World Health Organization. Antenatal Care randomized trial: manual for the implementation of the new model. Geneva: World Health Organization; 2002.

2. Ministério da Saúde. Assistência pré-natal: manual técnico da Secretaria de Políticas de Saúde. 3a Ed. Brasília: Ministério da Saúde; 2000.

3. Pender NJ, Murdaugh CL, Parsons MA. Health promotion in nursing practice. In: Pender NJ, Murdaugh CL, Parsons MA, editors. Upper saddle river. $5^{\text {th }}$ Ed. New Jersey: Pearson Education Inc; 2005.

4. Chamberlain G. Manual de assistência pré-natal. São Paulo: Livraria Santos Editora; 1993.

5. Lin YH, Tsai EM, Chan TF, Chou FH, Lin YL. Health Promoting Lifestyles and Related Factors in Pregnant Women. Chang Gung Med J 2009; 32:650-61.

6. Ministério da Saúde. Pré-natal e peruerpério atenção qualificada e humanizada: manual técnico. 3a Ed. Brasília: Ministério da Saúde; 2006. (Série A. Normas e Manuais Técnicos).

\section{Acknowledgements}

This research received support from $\mathrm{CNPq}$ and FAPERJ.
7. Szwarcwald CL, Mendonça MHM, Andrade CLT. Indicadores de atenção básica em quatro municípios do Estado do Rio de Janeiro, 2005: resultados de inquérito domiciliar de base populacional. Ciênc Saúde coletiva 2006 11:643-55.

8. Leal MC, Gama SG, Ratto KM, Cunha CB. Uso do índice de Kotelchuck modificado na avaliação da assistência pré-natal e sua relação com as características maternas e o peso do recém-nascido no Município do Rio de Janeiro. Cad Saúde Pública 2004; 20 Suppl 1:S63-72.

9. Coimbra LC, Silva AA, Mochel EG, Alves MT, Ribeiro VS, Aragão VM, et al. Factors associated with inadequacy of prenatal care utilization. Rev Saúde Pública 2003; 37:456-62.

10. Bassani DG, Surkan PJ, Olinto MT. Inadequate use of prenatal services among Brazilian women: the role of maternal characteristics. Int Perspect Sex Reprod Health 2009; 35:15-20.

11. Kawachi I, Subramanian SV, Kim D. Social capital and health. New York: Springer; 2008. 
12. Bourdieu P. The forms of social capital. In: Richardson JG, editor. Handbook of theory and research for sociology of education. New York: Greenwood Press; 1986. p. 241-8.

13. Putnam RD. Making democracy work: civic traditions in Modern Italy. Princeton: Princeton University Press; 1993.

14. Subramanian SV, Lochner KA, Kawachi I. Neighborhood differences in social capital: a compositional artifact or a contextual construct? Health Place 2003; 9:33-44.

15. Macintyre S, Ellaway A. 2000. Ecological approaches: rediscovering the role of the physical and social environment. In: Berkman LF, Kawachi I, editors. Social epidemiology. New York: Oxford University Press; 2000. p. 332-48.

16. Berkman LF, Kawachi I. Social epidemiology. New York: Oxford University Press; 2000.

17. Lia-Hoagberg B, Schaffer M, Strohschein S. Public health nursing practice guidelines: an evaluation of dissemination and use. Public Health Nurs 1999; 16:397-404.

18. Quelopana AM, Champion JD, Salazar BC. Factors predicting the initiation of prenatal care in Mexican women. Midwifery 2009; 25:277-85.

19. Wilkinson R, Pickett K. The spirit level: why more equal societies almost always do better. $1^{\text {st }} \mathrm{Ed}$. London: Penguin Group; 2009.

20. Kotelchuck M. An evaluation of Kessner adequacy of prenantal care index and a proposed adequacy prenantal care utilization index. Am J Public Health 1994; 84:1414-20.

21. Woolcock M, Narayan D. Social capital: implications for development theory, research and policy. The World Bank Research Observer 2000; 15: 225-49.

22. Pattussi MP. Neighbourhood social capital and oral health in adolescents [Doctoral Dissertation]. London: University College London; 2004.

23. Sampson RJ, Raudenbush SW, Earls F. Neighborhoods and violent crime: a multilevel study of collective efficacy. Science 1997; 277:918-24.

24. Miles-doan R. "Empowering” women. Network 1994; 15:14-6.

25. Muntaner C, Oates GL, Lynch JW. Social class and social cohesion: a content validity analysis using a nonrecursive structural equation model. Ann N Y Acad Sci 1999; 896:409-13.
26. Kennedy BP, Kawachi I, Prothrow-Stith D, Lochner K, Gupta V. Social capital, income inequality, and firearm violent crime. Soc Sci Med 1998; 47:7-17.

27. Caplan G. Support systems and community mental health. New York: Behavioral Publications; 1974.

28. Berkman LF, Syme SL. Social networks, host resistance, and mortality: a nine-year follow-up study of Alameda County residents. Am J Epidemiol 1979; 109:186-204.

29. Sherbourne CD, Stewart AL. The MOS social support survey. Soc Sci Med 1991; 38:705-14.

30. Griep RH, Chor D, Faerstein E, Lopes C. Apoio social: confiabilidade teste-reteste de escala no Estudo Pró-Saúde. Cad Saúde Pública 2003; 19:625-34.

31. Chor D, Griep RH, Lopes C, Faerstein, E. Medidas de rede e apoio social no Estudo Pró-Saúde: prétestes e estudo piloto. Cad Saúde Pública 2001; 17:887-96.

32. Associação Nacional de Empresas de Pesquisa. Critério de classificação econômica no Brasil. São Paulo: Associação Nacional de Empresas de Pesquisa; 1997.

33. Poortinga W. Social capital: an individual or collective resource for health? Soc Sci Med 2006; 62:292302.

34. Hendryx MS, Ahern MM, Lovrich NP, McCurdy AH. Access to health care and community social capital. Health Serv Res 2002; 37:87-103.

35. Leal MC, Gama SGN, Frias P, Szwarcwald CL. Healthy lifestyles and access to periodic health exams among Brazilian women. Cad Saúde Pública 2005; 21 Suppl:S78-88.

36. Lima-Costa MF, Matos DL. Prevalência e fatores associados à realização da amografia na faixa etária de 50-69 anos: um estudo baseado na Pesquisa Nacional por Amostra de Domicílios (2003). Cad Saúde Pública 2007; 23:1665-73.

37. St Clair PA, Smeriglio VL, Alexander CS, Celentano DD. Social network structure and prenatal care utilization. Med Care 1989; 27:823-32.

38. Fukuyama F. Trust: the social virtues and the creation of prosperity. New York: Free Press; 1995.

39. Marin A, Piccinini CA. Famílias uniparentais: a mãe solteira na literature. Psico (Porto Alegre) 2009; 40:422-9.

Submitted on 15/Jun/2010

Final version resubmitted on $05 /$ Nov/2010 Approved on 09/Nov/2010 